\title{
Towards Better Understanding Maker Ecosystems
}

\author{
James Fey \\ Katherine Isbister \\ jfey@ucsc.edu \\ kisbiste@ucsc.edu \\ University of California Santa Cruz \\ Santa Cruz, California, USA
}

\begin{abstract}
This paper presents early work towards the development of a set of lenses for analyzing Maker ecosystems. By ecosystems, we mean the combination of hardware offered and web-presence that teaches/sells/builds community around that hardware, as well as the community of makers themselves. We analyzed 35 ecosystems, including well-known larger ones, as well as smaller, specialized environments. We present a set of key factors for consideration by anyone intending to use an ecosystem for a particular population or need in research, along with a set of examples of how these lenses can be applied for analysis. Key factors include: whether the ecosystem is open or closed, how they present onboarding materials that help users learn the platform, whether there is a growth path that supports continued learning and development, what they require or expect from users to participate, and the avenues of community development available for makers to share their work and knowledge.
\end{abstract}

\section{CCS CONCEPTS}

- Human-centered computing $\rightarrow$ Human computer interaction (HCI).

\section{KEYWORDS}

Maker Movement, Communities of Practice, Maker Communities, Maker Ecosystems

\section{ACM Reference Format:}

James Fey and Katherine Isbister. 2021. Towards Better Understanding Maker Ecosystems. In FabLearn Europe / MakeEd 2021 - An International Conference on Computing, Design and Making in Education (FabLearn Europe / MakeEd 2021), June 2-3, 2021, St. Gallen, Switzerland. ACM, New York, NY, USA, 4 pages. https://doi.org/10.1145/3466725.3466760

\section{INTRODUCTION}

The research reported in this paper is aimed at providing a series of lenses for evaluating entities in the broader Maker field. In an initial look through maker product sites, it became apparent that limiting to a platform view didn't take into account the amount of overlap in different maker resources or communities of practice that aren't

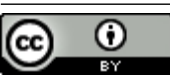

This work is licensed under a Creative Commons Attribution International 4.0 License.

FabLearn Europe / MakeEd 2021, June 2-3, 2021, St. Gallen, Switzerland

(c) 2021 Copyright held by the owner/author(s).

ACM ISBN 978-1-4503-8989-1/21/06.

https://doi.org/10.1145/3466725.3466760 directly tied to a particular platform. The constant shifting nature of maker resources with deprecating components and new hardware families developing, combined with overlapping permeable borders of these online entities, led to us taking an ecosystem approach to viewing the broader maker landscape. We focused specifically on what we refer to as Maker ecosystems-combinations of hardware offerings and accompanying web presence that provide teaching and community-building functions. These ecosystems do not have to be tied to a particular product line, but instead include the common materials that these communities of practice use. We drew upon the concept of "ecosystem-as-affiliation", which sees ecosystems as communities of associated actors defined by their networks and platform affiliations [Adner 2017] Within each maker ecosystem, one can find some subset of hardware sources, tutorials and project inspiration, a path for individual growth, and a community of other Makers sharing advice and their work. These ecosystems offer an on-ramp to a variety of technical areas such as computer engineering. Their use as informal and hands-on methods of learning are well documented and expanding. For example, O'Brien et al. and Jin et al. have both incorporated Maker methodology and hardware into educational settings [O'Brien et al. 2016][Jin et al. 2018].

\section{RELATED WORK}

Mellis and Buechley refer to the concept of ecosystems in relation to DIY (do-it-yourself), primarily from a fabrication and logistics standpoint [Mellis and Buechley 2014]. This was in the context of testing the technical edge of what DIY hardware affords to amateur users. Their term "open ecosystem" refers to open source hardware. Having open source hardware can aid in the development of components that work with first party hardware, but our terminology of open vs closed ecosystems refers instead to whether components from outside an ecosystem are compatible with a hardware platform, as well as whether an ecosystem affords makers an on-ramp to other connected ecosystems. Their use of the term also doesn't explore the educational materials or social structures of different ecosystems. Valverde-Berrocoso and Sánchez created a framework for analyzing Maker communities and the ecosystems they exist in through the the perspectives of trialogical learning: elements, interactions, and products [Valverde-Berrocoso and Fernández-Sánchez 2020]. Their approach aims to analyze how members of online maker communities learn through online forums and message boards as well as the issues these communities address. Kuznetov and Paulos conducted a survey of broader DIY communities, focused on how and why people contributed to them. They identify similar cultural values across Maker communities 
of what they dub "expert amateurs," who focus on personal skill development and sentiments of "anti-consumerism, rebelliousness, and creativity" [Kuznetsov and Paulos 2010]. Connected Learning Research Network promotes a more equitable set of entry points and pathways to educational, economic, and political opportunity [Ito et al. 2013]. They point out that many previous attempts to utilize technology to improve education focused narrowly on the deployment of particular media or technology without considering the broader contexts this learning exists in. Their ecology approach can be a useful lens for researchers studying making in education, encouraging a more holistic approach to understanding how making affects learner opportunities. However, there has not been, to our knowledge, exploration of how different Maker ecosystems within the wider Maker movement may foster individual skills and development. We aim to address this gap through landscape survey of Maker ecosystems ourselves to provide benefit to others who may be doing similar legwork for their own projects.

\section{METHOD}

We surveyed a total of 35 maker ecosystems, selected by gathering popular press articles about Maker culture, top ten lists of Maker kits and websites, and by asking five Maker educators about which ecosystems they use. We began with an initial set of 10 maker product pages, looking for commonalities in features such as tutorials and community content. From there we identified common traits in how they present their educational material, their onboarding material and how community created content supported officially produced educational resources. This led us to expand beyond maker product sites into community driven sites such as instructables, for a total of 35 ecosystems.

We used a grounded-theory approach to analyze the ecosystems [Wicks 2017]. We developed our set of five key factors through close analysis of the first 10 maker product pages, and refined them when analyzing the larger 35-ecosystem dataset against these factors. For each ecosystem, we systematically examined all major subareas of the website and related links. We kept a spreadsheet with notes about each ecosystem's resources pertaining to the factors. There is not enough room here to present a table of all of the ecosystems and their characteristics, a complete list is available upon request. Instead, in Section 5, we provide several examples to give a sense of how the set of lenses can be used.

\section{LENSES FOR ANALYZING MAKER ECOSYSTEMS}

Open vs. Closed Ecosystems: The factor of Open vs. Closed ecosystems addresses questions relating to whether or not an ecosystem affords the use of components outside of a product line of hardware. Are makers able to use components from other manufacturers? Are the items open source? Are there tutorials or guides for this hardware available outside of the manufacturer's website? Is there an on-ramp to other ecosystems? This is separate from growth path, as closed systems may still allow a high degree of growth and creativity for their users within their own hardware, but not allow for expansion outside this. This categorization can exist on a spectrum where an ecosystem may heavily feature tutorials for their own hardware, while still including lessons for generic electrical concepts, such as capacitance, or general skill tutorials, such as soldering or wiring. The Arduino ecosystem is characterized as being more open due to the array of compatible components, while LittleBits is comparatively more closed due to its heavy use of bespoke magnetic connectors. Educational Materials: This factor addresses questions relating to what resources are available within an ecosystem, and how are they presented. Are there projects produced by the manufacturer? Is there a set curriculum with a planned skill progression? What is the variety of project types featured? Are there resources to aid in curriculum creation for instructors? Some ecosystems have a curriculum of tutorials and projects that walk users through incrementally more complex topics. Others simply present users with a wide range of projects they could build. This, again, exists on a spectrum of unstructured projects to rigid curriculum. There might be a large collection of unrelated projects, with instances of smaller scale curriculum aimed at particular projects or hardware, which is the case for Instructables. This may be more useful for students looking to explore the possibilities of a domain space independently. Chibitronics presents a curriculum focused on teaching how electronics and circuits function in a structured way, moving from a single circuit to constructing a simple handmade sensor and beyond. This gives the user a base tool set of knowledge and techniques before moving to independent making. It provides a structured guiding hand that relaxes as the user gains skills and confidence. This works well if the goal is to move from skills to building and would be a well suited option if the goal is a more guided introduction to making. Barriers to Entry: This factor addresses questions pertaining to what an ecosystem asks of potential learners before they are able to meaningfully learn and create within it. What preexisting skills does this ecosystem require? What tools are required to create with this hardware? What is the economic cost of materials and are they reusable? Ecosystems have certain skill, material and financial cost in order to participate. For example, an ecosystem which heavily requires soldering would be inaccessible if the learner didn't have access to the tools or space required for fabrication. As we mention below, Make:Community is relatively accessible from a variety of skill levels but a wide array of projects require specialized tools and materials. Growth Path: This factor addresses questions relating to the direction and depth of skill and creative development afforded by an ecosystem. What skills are imparted by the tutorials for this ecosystem? Once a learner has completed an ecosystem's beginner kit, where are they directed to continue their development? Makers can reach a dead end if an ecosystem doesn't support continued growth of creativity and skill. If the goal is to have a user continue down a particular learning path without changing format or ecosystems, then a deeper progression of skills would be beneficial. If the goal is for a much shorter term experience to increase interest in making, then a short polished ecosystem may be preferred. A number of ecosystems feature tags on their tutorials for difficulty or subject matter. Makezine, Instructables, Hackerster and many others offer a surface level descriptor of beginner to advanced, which provides some guidance and context. Content can come from different sources. Maker ecosystems such as Elenco, MyNatureWatch, or Thingverse rely on their own community to support continued growth. Offering a place for users to showcase work and discuss with other Makers serves to keep engagement 
within an ecosystem going long after a user finishes with the set of official lessons or projects. Kiwico, on the other hand, has a growth path structured with a business model of being a subscription service. The path within their ecosystem is rigidly defined and, while users would need to look elsewhere to explore at their own pace, this offers a clear set of learning goals and a structured progression. Social Structures: Related to the idea of community-supported growth, this factor addresses questions pertaining to how makers within an ecosystem share their work and expertise. Can a user post a tutorial in a location easily viewable by others working within the ecosystem? Is there a place for makers to share questions and answers? Is there a mechanism for sharing work with others for critique and community building? This was A social media presence, forum, or blog that allows for makers within an ecosystem to interact can assist in creating computational communities[Kafai 2016]. This can aid in motivating continued engagement within an ecosystem, as well as allow makers to self sustain an ecosystem with new project ideas and guides. This generally requires the participation of the manufacturers and parts suppliers of the hardware that the ecosystem is built around. While there may be an independent community of makers focused on a specific ecosystem, if that community is supported and recognized by manufacturers and parts suppliers within the ecosystem, it raises the profile and directs new learners to preexisting communities of practice.

\section{EXAMPLE ECOSYSTEMS CATEGORIZED USING THE SET OF LENSES}

\subsection{Make:Community}

(https://make.co/) The Make:Community ecosystem functions as a hub in the overall Maker movement. While they hold physical events such as Maker Faires, Makezine functions primarily as a publisher of print and digital news and tutorials, while Make:Community serves to connect disparate groups of makers. Rather than producing or selling their own hardware, their tutorial guides deal with a wide array of hardware. Analysis: Open vs. Closed: They take an open approach towards the larger maker movement. While they don't manufacture their own hardware, they do provide connections to those that do. Their interactive board guide is useful for providing an overview of potential options. This is one of a number of meta resources they offer. Educational Resources: In addition to their platform specific publications, Make:Projects offers a collection of projects grouped by topic including Craft \& Design, Digital Fabrication, Science, Drones and Vehicles, Technology, Home and Workshop. Their online presence doesn't highlight curated curriculum. Growth Path: While there exists a strong ability to expand into a variety of subject areas, there isn't a structured progression of skill in Make:Community. Barriers to Entry: "Tested" projects are curated in the Makezine portion of the website, where projects are classified by time investment on a 4-point scale, and skill on a 3-point scale. The breadth of projects offers something accessible for everyone, but many of the project categories assume physical access to costly equipment at Maker spaces, such as 3D printing or laser cutting. Social Structures: In addition to their physical and social media presence acting as a nexus for Maker interaction, Make:Community offers a platform for makers to connect and share their work and interests.

\section{2 littleBits}

(https://sphero.com/collections/all/family_littlebits) Acquired by Sphero in 2019, the littleBits ecosystem is built around an electronics platform featuring magnetic connectors for components. This offers a very polished environment for learning and creating within the bounds of the ecosystem, but with very limited off ramps to further education. Analysis: Open vs. Closed: Closed, barring some recent hardware allowing for integration with the Micro:Bit ecosystem. Their bespoke magnetic connector limits the compatibility with outside components. Educational Resources: Each kit product has a set of activities and lessons pertaining to the theme of that kit. In the 'littleBits classroom' portion of their website, there are tools for educators to integrate different kits into technology curriculum. Growth Path: Very limited beyond the bounds of individual kits. There are further resources to expand learning into other ecosystems. Barriers to Entry: They list the skills required for each part, breaking it down to assembly, programming and electrical prototyping. Social Structures: The Sphero community page features a forum for sharing work and questions. Badge system encourages positive community interaction.

\subsection{Instructables}

(https://www.instructables.com/) Instructables is very project focused. It is an open ecosystem that relies on outside distributors for the materials required. Anyone can create an instructable, which makes it a good platform for posting a project or design for others to use. Analysis: Open vs. Closed: Open approach to tutorials. The guides featured here are not bound by a particular brand, and will sometimes include a blend of hardware from different ecosystems. Projects are grouped by themes rather than product lines. Educational Resources: The material available is dominated by unstructured, yet filterable, projects. There are a selection of projects organized into curriculum and a subsection of their work focuses on instructing teachers or providing resources. Growth Path: The depth of available content is very large, with approximately 84300 electronics tutorials at the time of writing. The example curriculum section of the ecosystem catalogs projects that have been featured and grouped by subject or school year. Barriers to Entry: While the typical Instructables format includes a list of materials and skills on the first page, there isn't a viable way to sort or filter by difficulty. Many projects are relatively low cost, but some require specialized tools and workshop space. Social Structures: They operate as a database of user-submitted instructions for a wide range of projects on topics from cooking to circuits.

\section{DISCUSSION}

\subsection{Living, Changing Ecosystems}

It is important to note that ecosystems may need to be revisited over time. Products that are key components of your process might be discontinued without notice. You might build a curriculum around a specific model of Sparkfun's Inventors Kit only to find that the contents of the kit change the following year. The process of creating educational resources requires an element of flexibility in order to adapt to the changing state of affairs across the network of Maker 
ecosystems. Just as any given ecosystem may change, the connections between ecosystems can ebb and flow. Engaging within this space requires flexibility and awareness. Specific product lines will rise and fall, affecting ecosystems that make use of them. On sites such as Adafruit and Sparkfun, you can still find documentation on retired products, but the components themselves are no longer available. Generally, there is a newer replacement version but there is no guarantee of this and they may no longer be compatible with the existing array of tutorials. You could be developing a design and decide to base it around the Intel Arduino 101 micro-controller only to receive word of Intel shutting down their Maker division. This set of lenses can provide a tool for continuous evaluation. If an educator or researcher needs to update a pre-existing curriculum, they could identify what ecosystem factors they are trying to match and use this perspective to evaluate different ecosystems to find one that meets their design criteria. It can serve as a method for evaluating design decision for maker education research. When trying to develop materials for maker education research, this set of lenses could serve to articulate design values to guide the development process.

\subsection{Ecosystems of Ecosystems}

While the open vs. closed lens seeks to help answer questions pertaining to hardware compatibility and the availability of instructional material, all of these ecosystems operate in a shared space and with that come connections and overlaps even for the most closed of ecosystems.

Just as Mellis and Buechley point out in their discussion of ecosystems, it is important to note that amateur making exists within the broader context of industry and supply chains that dictate which components or information are available [Mellis and Buechley 2014]. Similarly but from a more social perspective, larger meta ecosystems such as Instructables or Make:Community can serve as a context that smaller ecosystems share, which provides makers within those ecosystems a social platform on which to interact and learn from each other. Even a relatively closed ecosystem like Lego Mindstorms can share a connection with the BBC Micro:bit, for example, through the the Instructables ecosystem. The open vs. closed factor aims to articulate an ecosystem's relationship to its closest neighbors, to aid in the selection of an ecosystem that fits user needs.

\section{CONCLUSION}

In this paper we have presented the results of a landscape survey of Maker ecosystems in the form of a set of lenses for analysis. Important factors include: whether the ecosystem is open or closed, how they present onboarding materials that help users learn the platform, whether there is a growth path that supports continued learning and development, barriers to entry, and community structures. As we continue to develop these lenses we hope to gather feedback from the broader community of maker education research to refine this into a stronger contribution to the field. We hope that this will be useful to practitioners working within the Maker space, as well as provide a useful perspective for academics exploring the current state of the Maker field.

\section{ACKNOWLEDGMENTS}

This material is based upon work supported by the National Science Foundation under Grant No. 2005816.

\section{REFERENCES}

Ron Adner. 2017. Ecosystem as Structure. fournal of Management 43, 1 (1 2017), 39-58. https://doi.org/10.1177/0149206316678451

Mizuko Ito, Kris Gutiérrez, Sonia Livingstone, Bill Penuel, Jean Rhodes, Katie Salor, Juliet Schor, Julian Sefton-Green, and S. Craig Watkins. 2013. Connected Learning An Agenda for Research and Design. Digital Media and Learning Research Hub, Irvine, CA. 99 pages.

Karen H. Jin, Howard Eglowstein, and Mihaela Sabin. 2018. Using physical computing projects in teaching introductory programming. SIGITE 2018 - Proceedings of the 19th Annual SIG Conference on Information Technology Education 19 (2018), 155. https://doi.org/10.1145/3241815.3241879

Yasmin B. Kafai. 2016. From computational thinking to computational participation in K-12 education. Commun. ACM 59, 8 (8 2016), 26-27. https://doi.org/10.1145/ 2955114

Stacey Kuznetsov and Eric Paulos. 2010. Rise of the expert amateur: DIY projects, communities, and cultures. NordiCHI 2010: Extending Boundaries - Proceedings of the 6th Nordic Conference on Human-Computer Interaction 6 (2010), 295-304. https://doi.org/10.1145/1868914.1868950

David A. Mellis and Leah Buechley. 2014. Do-it-yourself cellphones: An investigation into the possibilities and limits of high-tech DIY. In Conference on Human Factors in Computing Systems - Proceedings. Association for Computing Machinery, New York, NY, USA, 1723-1732. https://doi.org/10.1145/2556288.2557309

Sean O'Brien, Alexandria K. Hansen, and Danielle B. Harlow. 2016. Educating teachers for the maker movement: Pre-service teachers' experiences facilitating maker activities. ACM International Conference Proceeding Series 0 (2016), 99-102. https: //doi.org/10.1145/3003397.3003414

Jesús Valverde-Berrocoso and María Rosa Fernández-Sánchez. 2020. Trialogical learning as a theoretical framework in the digital ecosystem of a maker community: A case study. Pedagogika 139, 3 (2020), 199-218. https://doi.org/10.15823/p.2020.139. 10

David Wicks. 2017. The Coding Manual for Qualitative Researchers (3rd edition) (3rd edition ed.). Vol. 12. SAGE, London. 169-170 pages. https://doi.org/10.1108/qrom08-2016-1408 